\title{
Ring polymer molecular dynamics beyond the linear response regime: Excess electron injection and trapping in liquids
}

\author{
Artur R. Menzeleev and Thomas F. Miller III \\ Division of Chemistry and Chemical Engineering, California Institute of Technology, \\ Pasadena, California 91106, USA
}

(Received 2 November 2009; accepted 22 December 2009; published online 20 January 2010)

\begin{abstract}
Ring polymer molecular dynamics (RPMD) is used to directly simulate the injection and relaxation of excess electrons into supercritical helium fluid and ambient liquid water. A method for modulating the initial energy of the excess electron in the RPMD model is presented and used to study both low-energy (cold) and high-energy (hot) electron injections. For cold injection into both solvents, the RPMD model recovers electronically adiabatic dynamics with the excess electron in its ground state, whereas for hot electron injection, the model predicts slower relaxation dynamics associated with electronic transitions between solvent cavities. The analysis of solvent dynamics during electron localization reveals the formation of an outgoing solvent compression wave in helium that travels for over $2 \mathrm{~nm}$ and the delayed formation of water solvation shells on the timescale of $300 \mathrm{fs}$. Various system-size effects that are intrinsic to the simulation of excess electron injection are discussed. Comparison of the RPMD simulations with previous mixed quantum-classical dynamics simulations finds general agreement for both the mechanisms and timescales for electron localization, although the electron localization dynamics in the RPMD model is essentially completed within $400 \mathrm{fs}$ in helium and $150 \mathrm{fs}$ in water. (C) 2010 American Institute of Physics. [doi:10.1063/1.3292576]
\end{abstract}

\section{INTRODUCTION}

Excess electron injection and localization in liquids is a prototype for coupled electronic and nuclear dynamics and for solvent dynamics beyond the linear response regime. As such, this process has received both experimental ${ }^{1-15}$ and theoretical ${ }^{16-24}$ attention. A widely used method for simulating electron injection, as well as many other electronically nonadiabatic processes, is mixed quantum-classical dynamics (MQCD), ${ }^{16,25-28}$ in which the excess electron is evolved in the wavefunction representation and the solvent is evolved using classical molecular dynamics. Here, we consider the alternative use of ring polymer molecular dynamics $(\text { RPMD })^{29,30}$ to simulate the dynamics of excess electron injection into fluid helium and liquid water.

We recently put forward RPMD as a model for the direct simulation of quantum mechanical systems, and we demonstrated that this model accurately describes the dynamics of an excess electron in dense fluid helium near thermal equilibrium. ${ }^{31}$ RPMD, like centroid molecular dynamics (CMD) ${ }^{32-34}$ employs the path integral representation ${ }^{35,36}$ to yield an approximate classical molecular dynamics model for the real-time simulation of quantum dynamics. The model dynamics rigorously preserves the quantum Boltzmann distribution, ${ }^{37,38}$ it allows for the simulation of long dynamical trajectories, and it provides a consistent framework for simulating both quantum mechanical and classical mechanical degrees of freedom. In the current study, we employ RPMD to simulate excess electron injection, a process that involves dynamics far from equilibrium, and we compare the

${ }^{a)}$ Electronic mail: tfm@caltech.edu. mechanisms and timescales obtained from the RPMD model with those from previous MQCD simulations.

The remainder of the paper is organized as follows. In Sec. II, we describe our method for simulating the excess electron injection using RPMD. In Sec. III A we discuss the high-energy and low-energy injection of an excess electron into the supercritical helium fluid, a simple model for a dense classical liquid. In Sec. III B, we similarly discuss the injection of an excess electron into ambient liquid water. Concluding remarks are presented in Sec. IV.

\section{METHODS}

\section{A. RPMD}

A quantized excess electron is simulated in a classical solvent of either helium or water. For both cases, the RPMD equations of motion are ${ }^{29}$

$$
\begin{aligned}
\dot{\mathbf{v}}^{(\alpha)}= & \omega_{n}^{2}\left(\mathbf{q}^{(\alpha+1)}+\mathbf{q}^{(\alpha-1)}-2 \mathbf{q}^{(\alpha)}\right) \\
& -\frac{1}{m} \nabla_{\mathbf{q}^{(\alpha)}} U\left(\mathbf{q}^{(\alpha)}, \mathbf{Q}_{1}, \ldots, \mathbf{Q}_{N}\right), \quad \alpha=1, \ldots, n, \quad, \\
\dot{\mathbf{V}}_{j}= & -\frac{1}{n M_{j}} \sum_{\alpha=1}^{n} \nabla_{\mathbf{Q}_{j}} U\left(\mathbf{q}^{(\alpha)}, \mathbf{Q}_{1}, \ldots, \mathbf{Q}_{N}\right), \quad j=1, \ldots, N .
\end{aligned}
$$

Here, $n$ is the number of ring polymer beads for the quantized electron, $N$ is the number of atoms in the classical solvent, and $\mathbf{q}^{(\alpha)}$ and $\mathbf{Q}_{j}$ are the respective positions of the electron beads and solvent atoms, such that $\mathbf{q}^{(0)}=\mathbf{q}^{(n)}$. Similarly, $\mathbf{v}^{(\alpha)}$ and $\mathbf{V}_{j}$ are the respective velocities of the electron 
beads and solvent atoms, and $m$ and $M_{j}$ are the corresponding masses. The intrabead harmonic frequency is given by $\omega_{n}=n /(\beta \hbar)$, where $\beta$ is the reciprocal temperature. The notations $\{\mathbf{q}\}=\left\{\mathbf{q}^{(1)}, \ldots, \mathbf{q}^{(n)}\right\}$ and $\{\mathbf{Q}\}=\left\{\mathbf{Q}_{1}, \ldots, \mathbf{Q}_{N}\right\}$ will hereafter be used to describe the full set of ring polymer bead positions and solvent atom positions, respectively. Equations (1) and (2) generate a classical dynamics that we employ as a model for the real-time dynamics of the system. ${ }^{31}$

The system is described using a potential energy function, $U(\mathbf{q},\{\mathbf{Q}\})$, that is a sum of solvent-solvent, $U_{\mathrm{s}-\mathrm{s}}(\{\mathbf{Q}\})$, and electron-solvent, $U_{\mathrm{e}-\mathrm{s}}(\mathbf{q},\{\mathbf{Q}\})$, interactions. As in our previous study, ${ }^{31}$ we describe the electron-helium system using the interaction potentials adopted by Berne, Coker, and coworkers. ${ }^{39,40}$ For the case of an excess electron in water, we employed the simple point charge (SPC) rigid water model $^{41}$ and the Schnitker-Rossky psedopotential for the electron-water interactions. ${ }^{42}$ Although more recent waterelectron pseudopotentials have been developed, ${ }^{43-46}$ and issues related to the accuracy of the Schnitker-Rossky pseudopotential have been raised, ${ }^{47,48}$ our choice was made to aid comparison with previous simulations of electron injection. ${ }^{17-19}$

\section{B. RPMD model for electron injection}

Experimental electron injection can be achieved using photoionization of a solvent molecule or another donor species. ${ }^{1,2,13,49}$ This process can be theoretically modeled as the sudden introduction of an excess electron to a configuration of the neat fluid that is drawn from its equilibrium Boltzmann distribution at temperature $T,{ }^{18}$

$$
P_{T}(\{\mathbf{Q}\}) \propto \exp \left[-U_{\mathrm{s}-\mathrm{s}}(\{\mathbf{Q}\}) /\left(k_{\mathrm{B}} T\right)\right] .
$$

Previous MQCD simulation studies initialized the excess electron in Born-Oppenheimer states within a specified energy range, ${ }^{17,22}$ but the RPMD model represents the electron in the position basis rather than the basis of electronic states. Although it would be possible to project the distribution of electrons onto a specific energy eigenstate, we instead modulate the energy of the injected electron via its initial temperature. The excess electron and solvent are initially distributed according to

$$
P_{0}\left(\mathbf{q},\{\mathbf{Q}\} ; T, T^{\prime}\right)=P_{T}(\{\mathbf{Q}\}) P_{T^{\prime}}(\mathbf{q} \mid\{\mathbf{Q}\}),
$$

where the second term is a conditional probability distribution function for the electron at temperature $T^{\prime}$ subject to a given solvent configuration,

$P_{T^{\prime}}(\mathbf{q} \mid\{\mathbf{Q}\})=\frac{\int d \mathbf{q}^{(1)} \cdots d \mathbf{q}^{(n)} \delta(\mathbf{q}-\overline{\mathbf{q}}) \exp \left[S\left(\{\mathbf{q}\},\{\mathbf{Q}\} ; T^{\prime}\right)\right]}{\int d \mathbf{q}^{(1)} \cdots d \mathbf{q}^{(n)} \exp \left[S\left(\{\mathbf{q}\},\{\mathbf{Q}\} ; T^{\prime}\right)\right]}$.

Here, $S(\{\mathbf{q}\},\{\mathbf{Q}\} ; T)$ describes the action for the electron ring polymer coupled to the liquid at temperature $T,{ }^{50}$

$$
\begin{aligned}
& S(\{\mathbf{q}\},\{\mathbf{Q}\} ; T) \\
& \quad=-\frac{1}{n k_{\mathrm{B}} T} \sum_{\alpha=1}^{n}\left(\frac{1}{2} m \omega_{n}^{2}\left(\mathbf{q}^{(\alpha)}-\mathbf{q}^{(\alpha+1)}\right)^{2}+U\left(\mathbf{q}^{(\alpha)},\{\mathbf{Q}\}\right)\right),
\end{aligned}
$$

and $\overline{\mathbf{q}}=n^{-1} \sum_{\alpha=1}^{n} \mathbf{q}^{(\alpha)}$ is the position of the ring polymer centroid.

We consider initial distributions corresponding to both high-energy ("hot") and low-energy ("cold") electron injections. For hot electron injection, we choose $T^{\prime}$ to ensure that the Boltzmann distribution occupies a significant fraction of electronically excited states. For cold electron injection, we choose $T^{\prime}=T$, such that the excess electron is initialized at the same temperature as the neat liquid. Following previous simulations, ${ }^{16,18,21,22}$ we employ $T=309 \mathrm{~K}$ for the simulations with helium and $T=300 \mathrm{~K}$ for the simulations with water. For hot injection into both solvents, we employed $T^{\prime}$ $=5000 \mathrm{~K}$. For the potential energy functions employed here, these parameters ensure that cold electron injection leads to an initial excess electron population that is almost entirely in the ground state. However, for hot electron injection into helium, approximately $75 \%$ of the initial excess electron population occupies electronically excited states; for hot electron injection into water, approximately $85 \%$ of the population initially occupies electronically excited states.

The RPMD simulation protocol for electron injection is implemented as follows: (1) Representative configurations of the neat liquid at equilibrium are generated from long molecular dynamics trajectories at constant temperature $T$. Independent solvent configurations are sampled from these trajectories. (2) Keeping the solvent configuration fixed, the ring polymer for an excess electron is introduced to the system and equilibrated using molecular dynamics at constant temperature $T^{\prime}$. (3) Keeping both the solvent configuration and the centroid of the ring polymer fixed, the internal modes of the ring polymer are equilibrated at temperature $T$. This step is redundant, and thus skipped, for the cold electron injection simulations where $T=T^{\prime}$. (4) The combined electron-solvent dynamics are evolved according to the RPMD equations of motion in Eqs. (1) and (2). The initial velocities for the solvent atoms and the ring polymer beads are drawn from the Maxwell-Boltzmann distribution at temperature $T$.

Our model for electron injection is inspired by the path integral molecular dynamics work of Parrinello and Rahman, ${ }^{37}$ in which an excess electron localizes in a molten $\mathrm{KCl}$ salt. Of course, these earlier simulations were taken as a demonstration of the thermodynamic favorability of the localized electron, whereas we emphasize the ring polymer trajectory as a model for real-time dynamics. The details of our RPMD initialization protocol were chosen so that the ensemble of RPMD trajectories are equivalent in the shorttime limit to the ensemble of partially adiabatic CMD trajectories $^{51,52}$ initialized from the nonequilibrium distribution in Eq. (4). In the Appendix, we provide further justification for this protocol by demonstrating that it is consistent with the RPMD approximation for a Kubo-transformed correlation function for which the system is initialized in a nonequilibrium distribution. 


\section{One-electron energy eigenvalue calculations}

As a means of analyzing the dynamics of electron injection and localization, the excess electron energy levels and eigenfunctions were calculated for the time series of solvent configurations harvested from the RPMD trajectories. At each configuration, the one-electron eigenvalue problem was solved using the iterative-and-block Lanczos scheme put forward by Webster et al. ${ }^{16}$ It is emphasized that these eigenvalue calculations are not necessary for evolving the RPMD equations of motion; the time series of eigenvalues for the excess electron are extracted a posteriori from the completed trajectories.

\section{Simulation details}

Simulations were performed by implementing the RPMD method within the DL_POLY2 molecular dynamics package. ${ }^{53}$ The equations of motion were integrated using the velocity Verlet algorithm. ${ }^{54}$ As in previous RPMD applications, ${ }^{31,55-61}$ the ring polymer coordinates were updated at each timestep due to the forces arising from the solvent potential $\left(-\nabla_{\mathbf{q}^{\alpha}} U\left(\mathbf{q}^{\alpha}, \mathbf{Q}_{1}, \ldots, \mathbf{Q}_{\mathrm{N}}\right)\right)$ and due to the exact evolution of the purely harmonic portion of the ring polymer potential. The resulting integration algorithm is time reversible and symplectic. ${ }^{62}$

The helium-electron simulation employed periodic boundary conditions with a cubic simulation cell of sidelength of $L=26.47 \AA$. The system consists of 1000 helium atoms and a single ring polymer. These parameters correspond to a reduced solvent density of $\rho^{*}=0.9$. All interactions were truncated at $6.4 \AA$. As is discussed in Sec. III, additional simulations for the electron in helium were also employed using 4096 helium atoms in a cubic simulation box of side length $L=42.36 \AA$, which corresponds to the same helium density.

The water-electron simulation employed periodic boundary conditions with a cubic simulation cell of side length of $L=31.08 \AA$. The system consists of 1000 classical SPC water molecules and the ring polymer for a single excess electron. Short-ranged interactions were truncated and shifted with a cutoff distance of $9 \AA$. Long-range electrostatic interactions between water molecules were included via Ewald summation. Following previous simulations of the excess electron in water, ${ }^{17-19,63}$ electrostatic interactions between the electron and the water molecules were truncated and shifted, and rigid-body constraints for the water molecules were enforced via the RATTLE algorithm. ${ }^{64}$

Unless otherwise specified, the electron was represented in all simulations by a ring polymer of $n=1024$ beads, which has been shown to yield adequate convergence for the path integral discretization in both systems. ${ }^{40,65}$ The real-time RPMD trajectories were evolved with a timestep of 5 $\times 10^{-4}$ fs. For both hot and cold electron injections in both water and helium solvents, 100 injection trajectories (step 4 in the above protocol) were each evolved for 1 ps. The system configuration was recorded during the trajectories at every femtosecond. We note that by employing a classical treatment of the water and helium solvent, we avoid the pos- sibility of unphysical coupling between the electron ring polymer dynamics and internal ring polymer modes of the solvent degrees of freedom. ${ }^{61}$

The details for the equilibration of the injected electron (steps 2 and 3 in the above protocol) are as follows. For hot injection, step 2 was performed using an RPMD simulation of length 150 fs for the electron with fixed solvent positions. Step 3 was performed using an RPMD simulation of length $300 \mathrm{fs}$, fixing the solvent positions and the ring polymer centroid position. For cold injection, steps 2 and 3 were combined into a single RPMD trajectory of length 300 fs with fixed solvent positions. These equilibration runs were thermostatted by resampling the velocities from the MaxwellBoltzmann distribution every 3 fs. To more efficiently sample the ring polymer particle density for cold electron injection, the equilibration runs in step 2 were initialized with the ring polymer in the region of the large ground-state wavefunction density. As a convergence check, the lengths of the equilibration trajectories for both solvents were doubled, and it was confirmed that the average for the ring polymer radius of gyration in the equilibration trajectories was converged.

For the simulations in both solvents, the electronic energy eigenstates were calculated once every 2 fs using a uniform grid of $32 \times 32 \times 32$ points that spanned the simulation cell. The grid density and the interaction cutoffs are consistent with those employed in previous MQCD simulations of electron injection and photoexcitation. ${ }^{17,19,21,22,66,67}$ The iterative Lanczos calculation of the adiabatic eigenstates employed 650 Krylov vectors with the exponential transform parameter ( $\beta$ in Ref. 16) of 0.1. The block Lanczos correction used ten blocks of 16 vectors. For the case of an excess electron in water, the eigenvalue calculations employed a smooth spherical cutoff ${ }^{68}$ for all interactions between 7.5 and $8.0 \AA$, and for the eigenvalue calculations of an electron in helium, interactions were truncated and shifted at $6.4 \AA$. The analysis of Turi and Borgis ${ }^{43}$ indicates that these parameters lead to adequate convergence.

Finally, we note that for a small fraction of trajectories, our hot injection protocol led to configurations in which the electron ring polymer spans the entire periodic simulation cell. In one extreme case, a single periodic replica of the ring polymer was stretched between two different periodic replicas of a solvent cavity; this unphysical configuration was metastable on the timescale of picoseconds. To mitigate this system-size effect, we systematically discarded any trajectory in which a given solvent atom was within a distance $s$ of beads from two different periodic replicas of the ring polymer for a combined time of more than $20 \mathrm{fs}$. For helium, we used $s=3 \AA$, and for water, we used $s=4 \AA$. This led to the rejection of approximately $3 \%$ of trajectories for helium simulations and $1 \%$ for water. A larger simulation cell would naturally lead to an ensemble of trajectories that is less biased by this artifact.

\section{RESULTS AND DISCUSSION}

We employ the RPMD model to investigate electron injection and localization in dense molecular liquids. Both the 
short-time limit upon injection and the long-time limit following injection are well understood from a statistical perspective. ${ }^{69}$ The process by which the system responds after electron injection, however, involves additional challenges due to coupled electronic and nuclear dynamics on the subpicosecond timescale.

For the simulations in both water and helium, the electron is injected into a solvent configuration that is characteristic of the neat liquid. At short times, the electron experiences a rugged potential energy landscape with cavities that correspond to density fluctuations in the liquid. The electronic ground state is spatially localized in the disordered solvent environment, ${ }^{70}$ but higher excited states form a conduction band of extended electronic eigenstates. ${ }^{20,71}$

In the limit of long simulation times, the system equilibrates with the electron strongly localized, or trapped, in a cage of solvent molecules. ${ }^{69}$ The thermodynamic driving force for the trapping of the excess electron arises from a tradeoff between stabilizing the electronic eigenstates and the penalty of creating a solvent cavity that is large enough to confine the excess electron. The lowest energy electronic states correspond to a nearly spherical $s$-type state and up to three $p$-type states for the solvents considered here. ${ }^{17,20}$

The dynamics of electron injection in liquids involves large-scale solvent rearrangements coupled to the nonadiabatic dynamics of the excess electron. It is a benchmark for the simulation of coupled electronic and nuclear dynamics, following the seminal MQCD work of Coker, Berne, and coworkers $^{20-22}$ for an electron in helium and by Rossky, Friesner, and coworkers ${ }^{16-18}$ for an electron in water, and it remains a topical challenge for both theory and experiment. These earlier MQCD trajectories identified mechanisms and timescales for the localization dynamics of the excess electron that provide a basis for comparison with the RPMD model employed here.

\section{A. Injection of an excess electron into supercritical helium}

\section{From the perspective of the electron}

Figure 1 presents a typical RPMD trajectory for cold electron injection into the helium fluid. Figure 1(a) displays the time series for the ring polymer radius of gyration and the excess electron eigenspectrum. The radius of gyration, $R_{g}$, is calculated from the configuration of the ring polymer using

$$
R_{g}^{2}=\frac{1}{n} \sum_{\alpha=1}^{n}\left(\mathbf{q}^{(\alpha)}-\overline{\mathbf{q}}\right)^{2}
$$

Figure 1(b) shows snapshots of the ring polymer at various times along the injection trajectory, and Figs. 1(c) and 1(d) show the corresponding snapshots for the ground state $\left(\Psi_{0}\right)$ and first excited state $\left(\Psi_{1}\right)$ eigenfunctions. As was previously discussed, the trajectory is evolved according to the RPMD equations of motion, and the eigenstates for the excess electron are calculated afterwards for the purpose of analysis.

For cold electron injection, the ground state dominates the initial electron population. It is thus reasonable in Fig. 1

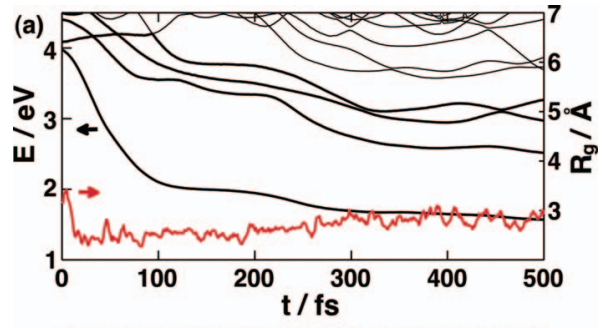

(b)

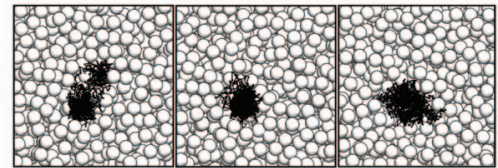

(c)

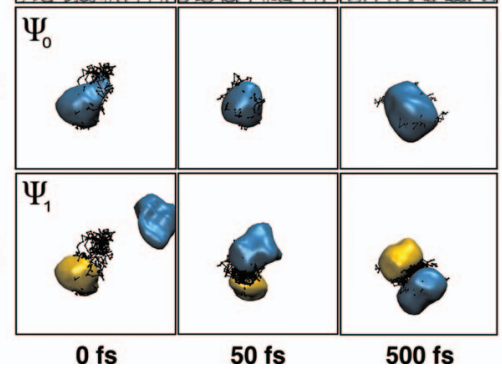

FIG. 1. A typical RPMD trajectory for cold electron injection into supercritical helium. (a) The time series for the lowest excess electron eigenenergies (black) and the ring polymer radius of gyration (red). (b) Snapshots of the ring polymer (black) and solvent configurations from the RPMD trajectory. [(c) and (d)] The corresponding snapshots of the ground state (c) and first excited state (d) excess electron wavefunctions, visualized as $95 \%$ isosurfaces.

that the ring polymer occupies the region of space for which the ground-state wavefunction is most probable. As the RPMD trajectory evolves in time, $R_{g}(t)$ for the ring polymer rapidly decreases and approaches the equilibrium average value of $2.6 \AA$. On a similar timescale, the solvent molecules rearrange to accommodate the excess electron in a solvent cavity. The formation of the solvent cavity is indicated by the decreasing energy of the lowest eigenstates that form the trapped states for the electron. The instantaneous configurations of the solvent cavity are not perfectly symmetrical, so the $p$-like excited states of the trapped electron are nondegenerate.

The majority of trajectories following cold electron injection are qualitatively similar to that shown in Fig. 1. To extract a statistically meaningful description, however, we consider the ensemble of RPMD trajectories for electron injection. In Fig. 2(a), we present the radius of gyration of the excess electron in fluid helium, $\left\langle R_{g}(t)\right\rangle$, where the angle brackets indicate the nonequilibrium average over RPMD trajectories at time $t$ after injection. Also shown in this figure is the contour plot for the distribution of $R_{g}(t)$ from the ensemble of trajectories. Both the average and the distribution exhibit behavior that is consistent with the trajectory in Fig. 1. The electron initially collapses into a nascent solvent cavity within $50 \mathrm{fs}$, the solvent cavity then expands so that $\left\langle R_{g}(t)\right\rangle$ exceeds the equilibrium value at approximately 300 fs, and the system subsequently relaxes toward equilibrium at longer times.

Hot electron injection exhibits an additional mechanism for localization. Along with the one-cavity localization pathway seen in cold electron injection, the hot injection trajec- 

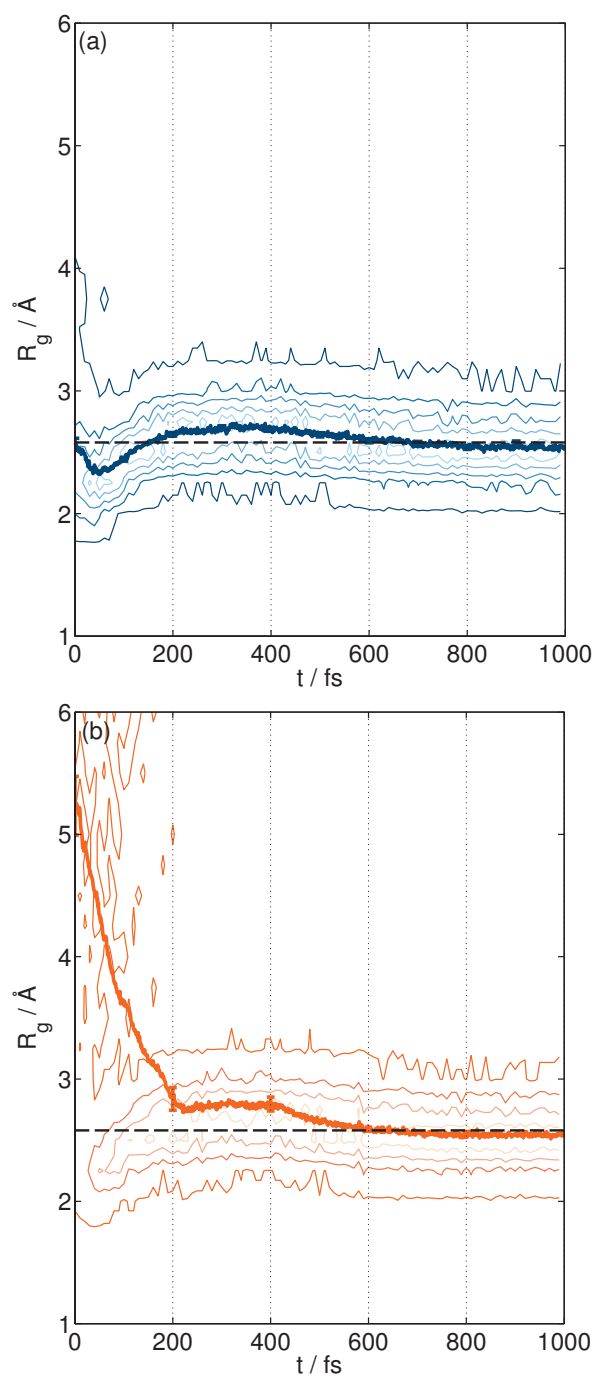

FIG. 2. Ring polymer radius of gyration following (a) cold injection and (b) hot injection of the excess electron into helium. The contour plot indicates the distribution of $R_{g}(t)$ from the ensemble of RPMD trajectories, the heavy colored lines indicate the nonequilibrium average taken over trajectories $\left\langle R_{g}(t)\right\rangle$, and the black dashed line indicates the average from an equilibrium simulation.

tories reveal that the electron transiently occupies multiple solvent cavities at the same time. Figure 3 presents one such trajectory. Figure $3(\mathrm{~b})$ shows that the ring polymer is initially extended in the solvent, and it collapses into a metastable, multicavity configuration within $50 \mathrm{fs}$. As the solvent undergoes further rearrangement, the ring polymer collapses into two distinct solvent cavities at $70 \mathrm{fs}$ and then a single cavity at approximately $200 \mathrm{fs}$. Figures 3(c) and 3(d) reveal that the ring polymer configuration is more consistent with the density of the first excited state wavefunction than the groundstate wavefunction at $50 \mathrm{fs}$ following injection.

This multicavity mechanism for localization following hot injection is further illustrated in Fig. 2(b). In a large fraction of the hot injection trajectories, the ring polymer fully localizes on the $50 \mathrm{fs}$ timescale, as was seen in cold injection. However, a significant number of trajectories exhibit extended ring polymer configurations that survive for hundreds of femtoseconds. The longest multicavity configurations persist for approximately $400 \mathrm{fs}$ in our ensemble of hot injection trajectories for helium.

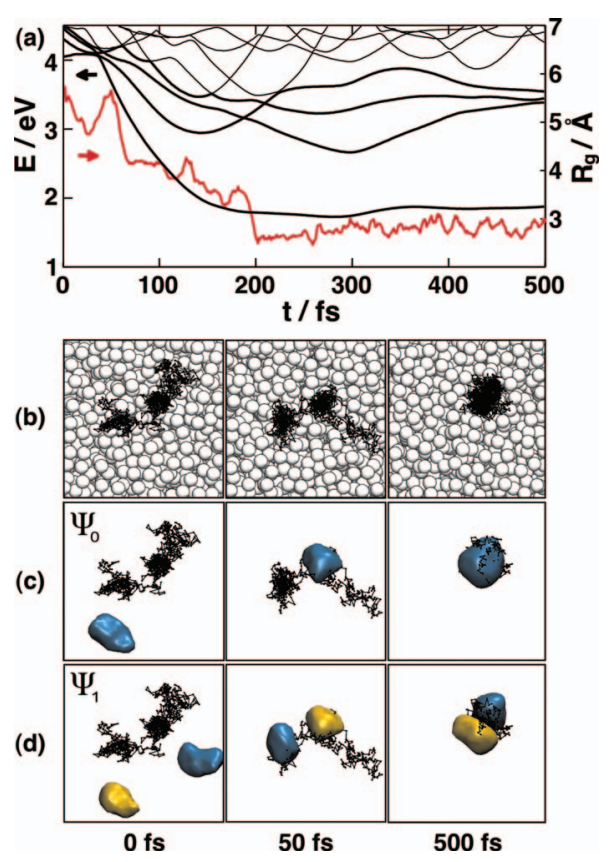

FIG. 3. A typical RPMD trajectory for hot electron injection into supercritical helium. (a) The time series for the lowest excess electron eigenenergies (black) and the ring polymer radius of gyration (red). (b) Snapshots of the ring polymer (black) and solvent configurations from the RPMD trajectory. [(c) and (d)] The corresponding snapshots of the ground state (c) and first excited state (d) excess electron wavefunctions.

\section{From the perspective of the solvent}

The solvent dynamics during electron localization was investigated by computing the time-resolved electron-helium radial distribution function, $\left\langle g_{\mathrm{e}-\mathrm{He}}(r, t)\right\rangle$, for the distance between the ring polymer centroid and the solvent helium atoms. Here, $g_{\mathrm{e}-\mathrm{He}}(r, t)$ refers to the radial distribution function from a single configuration of the system at time $t$ after injection, and the angle brackets indicate the nonequilibrium average over RPMD trajectories at time $t$. Figure 4(a) presents $\left\langle g_{\mathrm{e}-\mathrm{He}}(r, t)\right\rangle$ for cold electron injection. Even at very short times, the electron ring polymer is localized within a solvent cavity, as is seen by the depletion of helium atom density near the centroid of the ring polymer. However, the initial solvent cavity is smaller than at equilibrium, and it expands rapidly as the solvent atoms are expelled from the neighborhood of the electron. As is seen from the $80 \%$ contour line plotted in the figure, the solvent cavity expands to a radius of approximately $4.5 \AA$ by $200 \mathrm{fs}$, and this rapid expansion creates a compression wave in the helium fluid (indicated by the dashed line in the figure). The wave of high solvent density travels outward at $28 \AA / \mathrm{ps}$, reaching the edge of the periodic simulation cell by approximately $350 \mathrm{fs}$. The solvent cavity subsequently contracts to a radius of approximately $4 \AA$ at $800 \mathrm{fs}$, at which time a recurrence of high solvent density at the edge of the electron cavity is also observed. The origin of this interesting recurrence will be discussed below. The creation of compression waves during electron localization and solvent cavity expansion was previously explored in density functional theory calculations involving liquid helium at lower density and temperature. ${ }^{23} \mathrm{We}$ expect that solvent compression waves also appear in MQCD simulations of electron injection in helium, although 

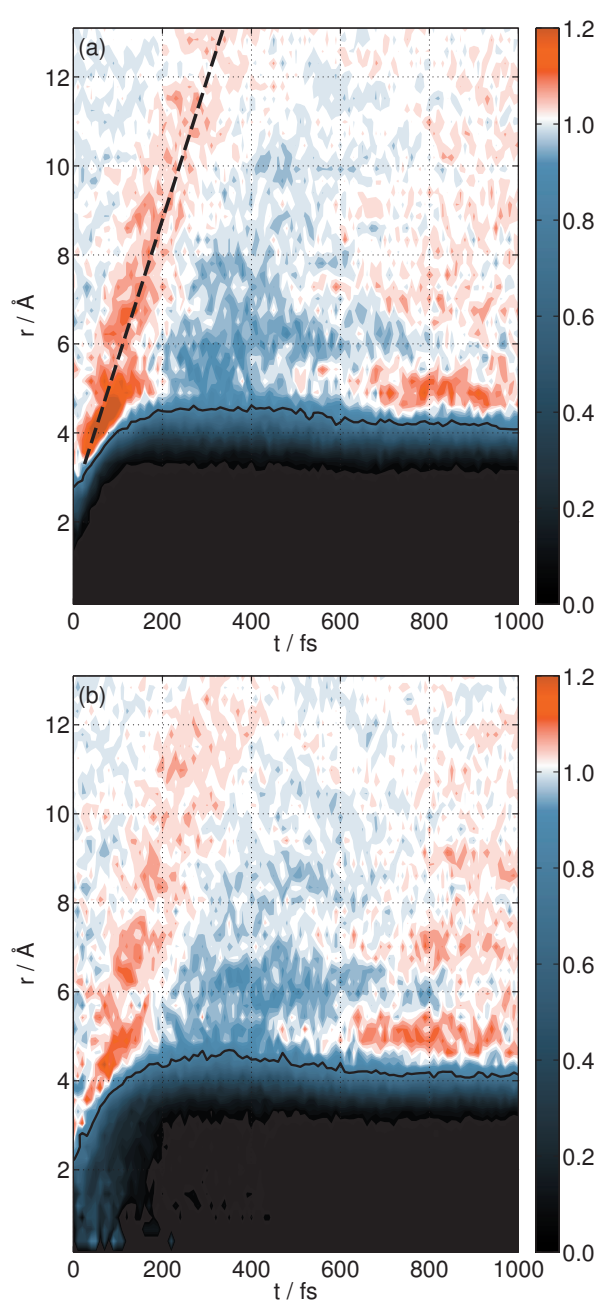

FIG. 4. Time-resolved radial distribution function $\left\langle g_{\mathrm{e}-\mathrm{He}}(r, t)\right\rangle$ for the helium atoms with respect to the electron ring polymer centroid for (a) cold injection and (b) hot injection. In both parts, the contour line indicates $80 \%$ of the average solvent density. The dashed line in part (a) indicates the outward solvent compression wave following electron localization.

to our knowledge, Fig. 4 presents the first report of these waves in a simulation with explicit solvent. The corresponding plot for hot electron injection [Fig. 4(b)] shows significant density near $r=0$ at short times following injection. Furthermore, the $80 \%$ contour line in Fig. 4(b) intercepts the $y$-axis at a considerably smaller value than in Fig. 4(a). These features indicate the presence of helium atoms near the centroid of the ring polymer due to trajectories in which the electron ring polymer initially spans multiple solvent cavities. By 200 fs, this feature has disappeared, which is consistent with the timescale for the ring polymer localization in Fig. 2(b). At longer times, solvent dynamics for hot injection are similar to those for cold electron injection.

The eigenenergies of the excess electron provide another means of analyzing the solvent dynamics. In Fig. 5, we present the excess electron energy eigenstates following both cold and hot electron injections, obtained from the nonequilibrium average over the ensemble of RPMD trajectories. As was seen in the individual trajectories in Figs. 1 and 3, the lowest states rapidly drop in energy as the electron localizes. Past $200 \mathrm{fs}$, the average eigenenergies oscillate on the same timescale as the solvent cavity size seen in Fig. 4. This rela-

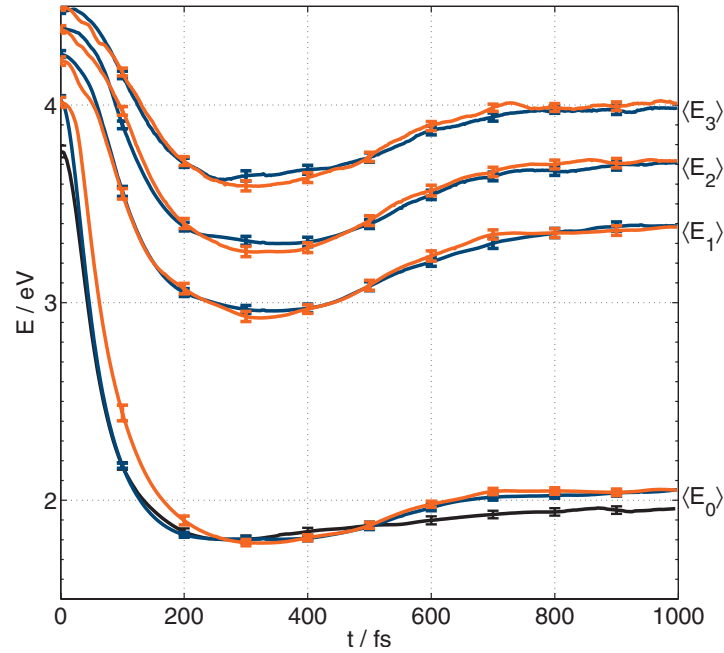

FIG. 5. The four lowest electron eigenenergies, obtained from the nonequilibrium average for RPMD trajectories following cold (blue) and hot (red) injection into the helium fluid. The black curve shows the corresponding ground-state result from the cold injection simulations with 4096 helium atoms. The black line exhibits a lower initial ground-state energy for the electron because the larger system size supports larger solvent density fluctuations in the neat fluid, even at the same average fluid density.

tionship between the electron energy levels and the size of the solvent cavity is expected from a simple square-well picture for the localized electron. Solvent cavity oscillations have been experimentally observed following electron cavity expansion in low-temperature, low-density liquid helium, ${ }^{72}$ but it is not obvious that such a large effect would survive in the more dense and viscous solvent regime considered here. ${ }^{23,24,72}$

At 800 fs following electron injection, both Figs. 4(a) and 4(b) exhibit a pronounced recurrence of solvent density at the edge of the solvent cavity. One possible explanation for this feature is that electron localization drives atoms in the first solvation shell of the electron to collide with atoms in the second shell and ballistically rebound, causing a recurrence of solvent density at the cavity edge. But it is also possible that this feature appears when the outgoing solvent compression wave from the electron reaches its periodic neighbor. (Note that at $28 \AA / \mathrm{ps}$, the solvent compression will begin arriving at the neighboring solvent cavity image at approximately $650 \mathrm{fs}$.) To determine whether the result is physically significant or a simulation artifact, we repeated the cold electron injection trajectories at the same solvent density but in a simulation cell for which the sidelength is increased by approximately $60 \%$.

In Fig. 6, we present the $\left\langle g_{\mathrm{e}-\mathrm{He}}(r, t)\right\rangle$ obtained from these larger simulations in helium. The initial solvent dynamics are similar to those found in the smaller cell. A solvent cavity rapidly expands, driven by the localization of the excess electron, and a solvent compression wave propagates outward from the cavity at the same rate. However, for the larger simulations, the oscillation in the cavity size is less dramatic, and the recurrence of large solvent density at the edge of the solvent cavity is significantly delayed in comparison to Fig. 4. This result suggests that while the outgoing compression wave is physically genuine, the pronounced recurrence of solvent density at the cavity edge is caused by 


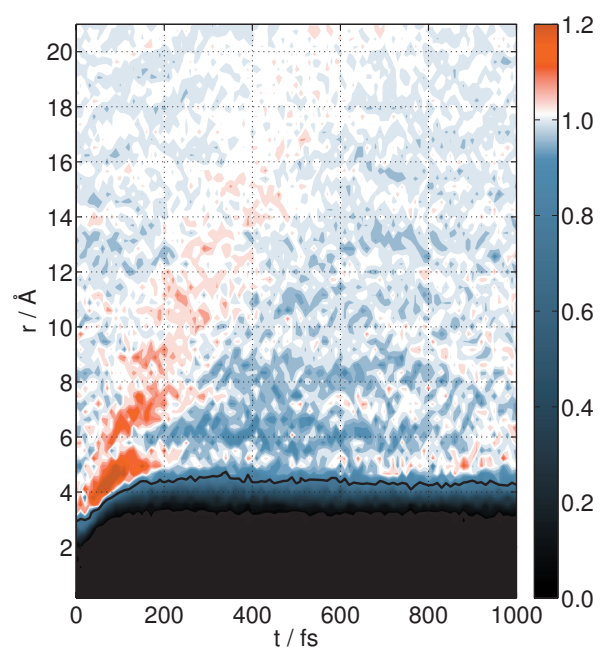

FIG. 6. Time-resolved radial distribution function $\left\langle g_{\mathrm{e}-\mathrm{He}}(r, t)\right\rangle$ following cold injection in the larger simulations with 4096 helium atoms, to be compared with Fig. 4(a).

the finite system size of the simulations. The conclusion is further supported by our calculation of the trajectoryaveraged electron ground-state energy for the larger system, plotted as a black line in Fig. 5. The rebound of the groundstate energy is much less dramatic at $800 \mathrm{fs}$ in the larger system because the compression wave from the neighboring periodic replica has not yet arrived. We emphasize that the system-size effect is not an artifact of the RPMD model, and we expect that it also appears in MQCD simulations.

The qualitative mechanisms and timescales observed in our RPMD simulations are similar to those reported previously in MQCD simulations of electron injection into helium. ${ }^{22}$ Injecting hot electrons at an energy of $0.5 \mathrm{eV}$ above the instantaneous ground-state energy, Space and Coker also found two distinct pathways for electron localization. For an ensemble of $15 \mathrm{MQCD}$ trajectories, most trajectories exhibited nonadiabatic electron relaxation of the electron to single-cavity state in 50-100 fs, followed by solvent cavity expansion over the next 100-200 fs. In a few trajectories, however, the electron was trapped in an excited state and remained in a two-cavity configuration for 300-400 fs before ultimately completing its relaxation in a single cavity. The total excited-state survival time for such "hung" trajectories was 600-700 fs. While we do observe a similar multicavity relaxation mechanism in the RPMD simulation, our timescale for the relaxation of these configurations is more rapid, with all 100 RPMD trajectories collapsing into a single cavity within 400 fs.

\section{Adiabatic versus nonadiabatic dynamics}

We employ an energy conservation measure to directly compare the RPMD model with adiabatic BornOppenheimer dynamics. Figure 7(a) presents various energy components for a typical cold injection RPMD trajectory in helium. The blue curve presents $K_{\mathrm{s}}(\{\mathbf{V}(t)\})+U_{\mathrm{s}-\mathrm{s}}(\{\mathbf{Q}(t)\})$, the sum of the solvent kinetic energy term, and the solventsolvent potential energy term that are obtained from the RPMD trajectory. Also plotted are the electronic eigenstates for the excess electron that are calculated from solvent con-
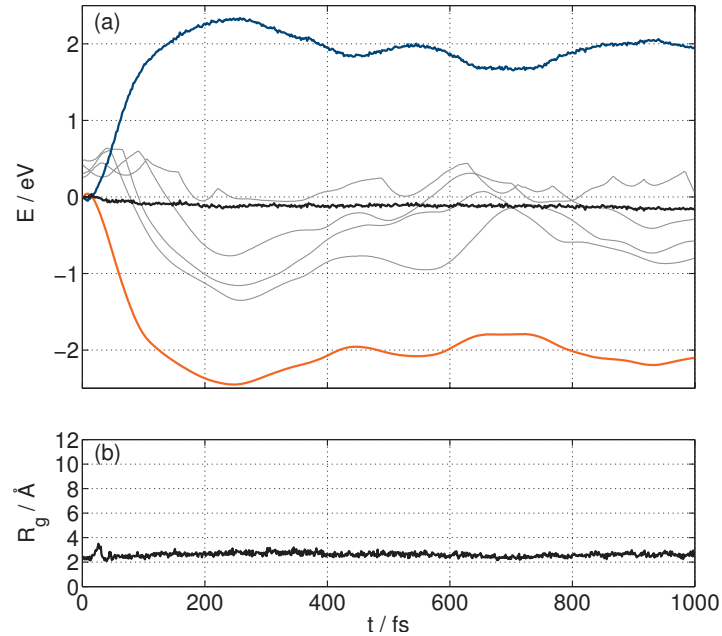

FIG. 7. An illustrative cold injection trajectory in helium, showing the time series for (a) various energy components and (b) the ring polymer radius of gyration. In part (a), the blue curve presents $K_{\mathrm{s}}(\{\mathbf{V}(t)\})+U_{\mathrm{s}-\mathrm{s}}(\{\mathbf{Q}(t)\})$, the sum of the solvent kinetic energy term and the solvent-solvent potential energy term, the red curve plots the electronc ground-state energy $E_{0}(\{\mathbf{Q}(t)\})$, the gray curve plots other low-lying electronic eigenenergies, and the heavy black curve plots the ground-state Born-Oppenheimer Hamiltonian, $H_{\mathrm{BO}}(t)=K_{\mathrm{s}}(\{\mathbf{V}(t)\})+U_{\mathrm{s}-\mathrm{s}}(\{\mathbf{Q}(t)\})+E_{0}(\{\mathbf{Q}(t)\})$. Energy components are shifted by constant values for graphical clarity.

figurations along the RPMD trajectory, with the red curve corresponding to the electron ground-state energy $E_{0}(\{\mathbf{Q}(t)\})$, and the gray curves corresponding to low-lying excited state energies. Finally, the black curve represents $H_{\mathrm{BO}}(t)$ $=K_{\mathrm{s}}(\{\mathbf{V}(t)\})+U_{\mathrm{s}-\mathrm{s}}(\{\mathbf{Q}(t)\})+E_{0}(\{\mathbf{Q}(t)\})$, which is the energy function that would be conserved if the trajectory evolved according to the ground-state Born-Oppenheimer Hamiltonian. In cold electron injection, as was earlier discussed, the initial population of the excess electron is dominated by the electronic ground state. The conservation of $H_{\mathrm{BO}}(t)$ in Fig. 7(a) indicates that the RPMD model is consistent with the adiabatic ground-state dynamics throughout the cold electron injection trajectory.

Figure 8 presents the corresponding analysis for a typical hot injection trajectory that exhibits the two-cavity localization pathway. At short times, the ground-state Born-
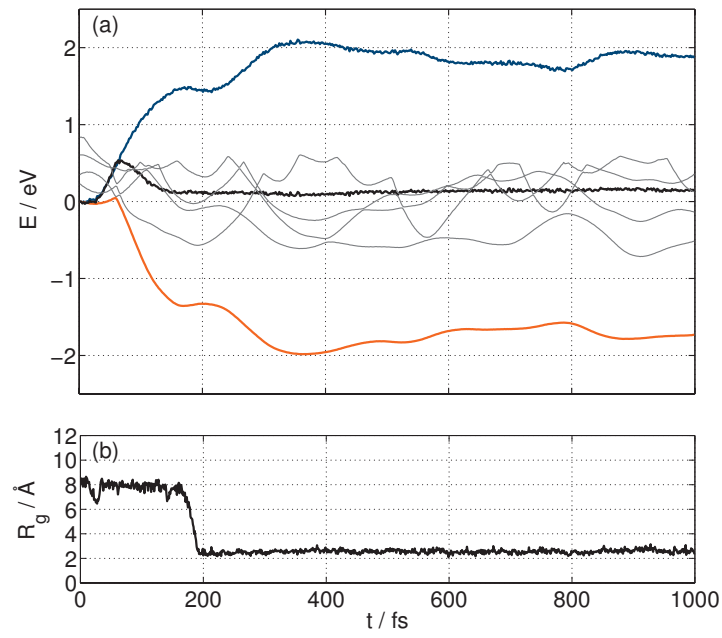

FIG. 8. An illustrative hot injection trajectory in helium, presented as in Fig. 7. 

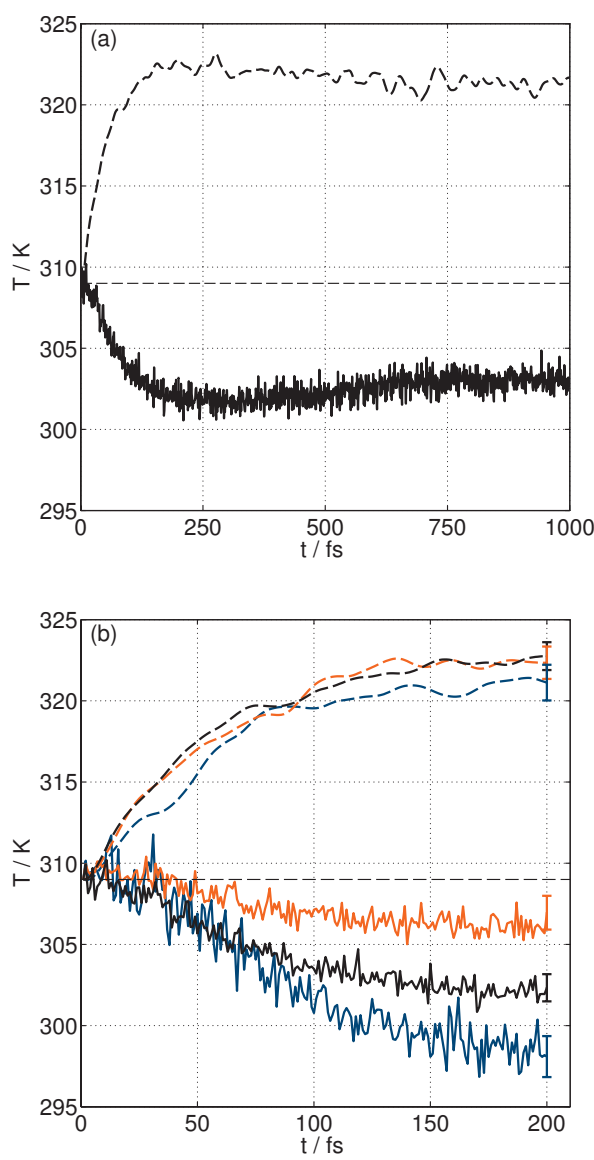

FIG. 9. The nonequilibrium average temperature for the helium solvent atoms (dashed) and for the ring polymer beads (solid) following cold electron injection. (a) Results obtained over 1 ps using 1024 ring polymer beads and (b) obtained for a shorter period using 512 (blue), 1024 (black), and 2048 (red) ring polymer beads. Error bars indicate the standard deviation of the mean.

Oppenheimer Hamiltonian is clearly not conserved along the RPMD trajectory. However, after the ring polymer localizes into a single solvent cavity at $200 \mathrm{fs}$ [Fig. 8(b)], then $H_{\mathrm{BO}}(t)$ becomes a constant of the motion. Although the RPMD model does not evolve the electron in its eigenstate representation, it clearly exhibits the transition from an initial excited-state population to ground-state dynamics as a function of time.

A more extensive analysis of the ensemble of RPMD trajectories for hot electron injection and cold electron injection yields results that are entirely consistent with the illustrative trajectories presented in Figs. 7 and 8.

\section{Energy dissipation and slow equilibration timescales}

Although the solvent bath of 1000 helium atoms might seem an ample reservoir for dissipating the energy associated with the electron injection and localization, Fig. 9(a) reveals that this is not the case. The dashed line in this figure presents the temperature of the solvent atoms following cold electron injection, obtained from the nonequilibrium average over the ensemble of RPMD trajectories. Initially, the solvent temperature is $309 \mathrm{~K}$, as is required by our injection protocol, but this temperature rises to over $320 \mathrm{~K}$ as the electron relaxes and forms a solvent cavity. This is yet another finite size effect that should be considered in simulations of electron injection. Given that the RPMD trajectories are essentially reproducing ground-state adiabatic dynamics for the case of cold electron injection [Fig. 7(a)], it is almost certain that this effect also appears in MQCD simulations (although we find that the greater heat capacity of water leads to a less pronounced increase in temperature than for helium).

The solid line in Fig. 9(a) shows the corresponding evolution of the temperature of the ring polymer beads, also obtained from the nonequilibrium average over RPMD trajectories. At short times, the temperature of the ring polymer beads drops as the electron adiabatically expands during the formation of the solvent cavity. However, even after a picosecond of dynamics, the ring polymer temperature remains very different from that of the solvent atoms. This slow equilibration timescale, which is a well known hindrance to the convergence of path integral molecular dynamics simulations, ${ }^{73}$ arises from the separation of timescales between the motions of the ring polymer beads and the solvent atoms. Interestingly, slow equilibration between the ring polymer and the solvent seems to be a necessary feature of the RPMD model's correct description of the adiabatic Born-Oppenheimer dynamics for cold electron injection [Fig. 7(a)]; if the internal modes of the ring polymer rapidly equilibrated with the solvent atoms, then the average solvent kinetic energy would have dropped and $H_{\mathrm{BO}}(t)$ would not have been a constant of the motion.

The dashed lines in Fig. 9(b) show that the calculated rise in solvent temperature following electron injection is independent of the number of ring polymer beads (in the limit that the bead number is converged with respect to the path integral discretization, of course). This is not surprising, but it does confirm that the coupling between the solvent and ring polymer dynamics does not significantly depend on this parameter. The solid lines in Fig. 9(b) also show that increasing the number of ring polymer beads diminishes the amount to which the time-evolved ring polymer temperature deviates from its initial value. This is also easily understood. The similarity of the dashed lines in Fig. 9(b) suggests that the amount of energy exchanged between the solvent and ring polymer beads during electron localization is independent of the number of beads. Increasing the number of beads, which undergo relatively fast equilibration with respect to each other, simply decreases the fraction of this fixed amount of energy that is withdrawn from the average energy of each bead.

We conclude this section by noting that if a sufficiently large solvent bath were employed, then the average rise in the solvent temperature in Fig. 9(a) would not have occurred. Also, if a sufficient number of ring polymer beads were employed, then the corresponding drop in the ring polymer temperature in Fig. 9(b) would not have been observed. These features, while interesting, are artifacts of our finite simulation size, rather than fundamental drawbacks of the RPMD model. 


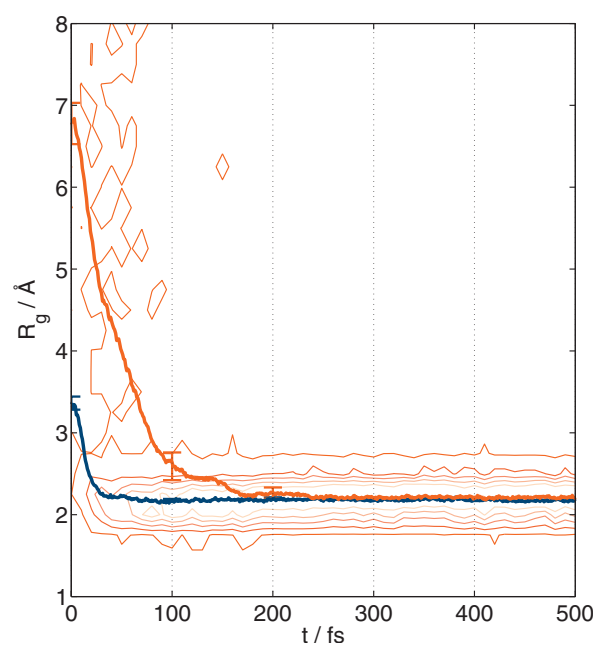

FIG. 10. Ring polymer radius of gyration following excess electron injection in water. The contour plot indicates the distribution of $R_{g}(t)$ from the ensemble RPMD trajectories for hot injection, the heavy lines indicate the nonequilibrium average $\left\langle R_{g}(t)\right\rangle$ taken over cold injection (blue) and hot injection (red) trajectories.

\section{B. Injection of an excess electron into liquid water}

Liquid water provides a more complex environment for electron injection than helium by introducing solvent hydrogen bonding and rotational motion. Nonetheless, many features of the water simulations will be familiar from our analysis of electron injection into helium.

Figure 10 presents the ring polymer radius of gyration following injection into liquid water. As was seen for helium, the ring polymer is initially more extended for the case of hot injection. The figure also shows that the hot injection trajectories in water exhibit a slower timescale for relaxation of the ring polymer than cold injection trajectories. For cold injection, $\left\langle R_{g}(t)\right\rangle$ approaches its equilibrium value within $30 \mathrm{fs}$, on the timescale of the librational motion of the water molecules. However, a fraction of the hot injection trajectories exhibit a slower relaxation pathway in which the ring polymer transiently occupies multiple solvent cavities. In general, we find that these multicavity configurations survive for less time in water than in helium, suggesting that the water solvent allows for more facile rearrangment of the electron solvation environment at short times.

To further investigate the dynamics of the solvent, Fig. 11 presents the electron-hydrogen radial distribution function $\left\langle g_{\mathrm{e}-\mathrm{H}}(r, t)\right\rangle$ and the electron-oxygen radial distribution function $\left\langle g_{\mathrm{e}-\mathrm{O}}(r, t)\right\rangle$ for cold electron injection; both are calculated in terms of the distance between the ring polymer centroid and the corresponding solvent atoms. As was the case in cold helium injection, the initial absence of both oxygen and hydrogen atoms near the electron centroid indicates that the injected electron occupies a presolvation environment that is depleted of water molecules. ${ }^{74,75}$ For times shorter than $100 \mathrm{fs}$ following injection, the primary solvent rearrangements correspond to the reorientation of the solvent hydrogen atoms in the vicinity of the electron. However, another significant solvent rearrangement occurs on the 300 fs timescale, as the first peak in $\left\langle g_{\mathrm{e}-\mathrm{O}}(r, t)\right\rangle$ bifurcates to form the first two distinct shells of the solvent cage. Also on this
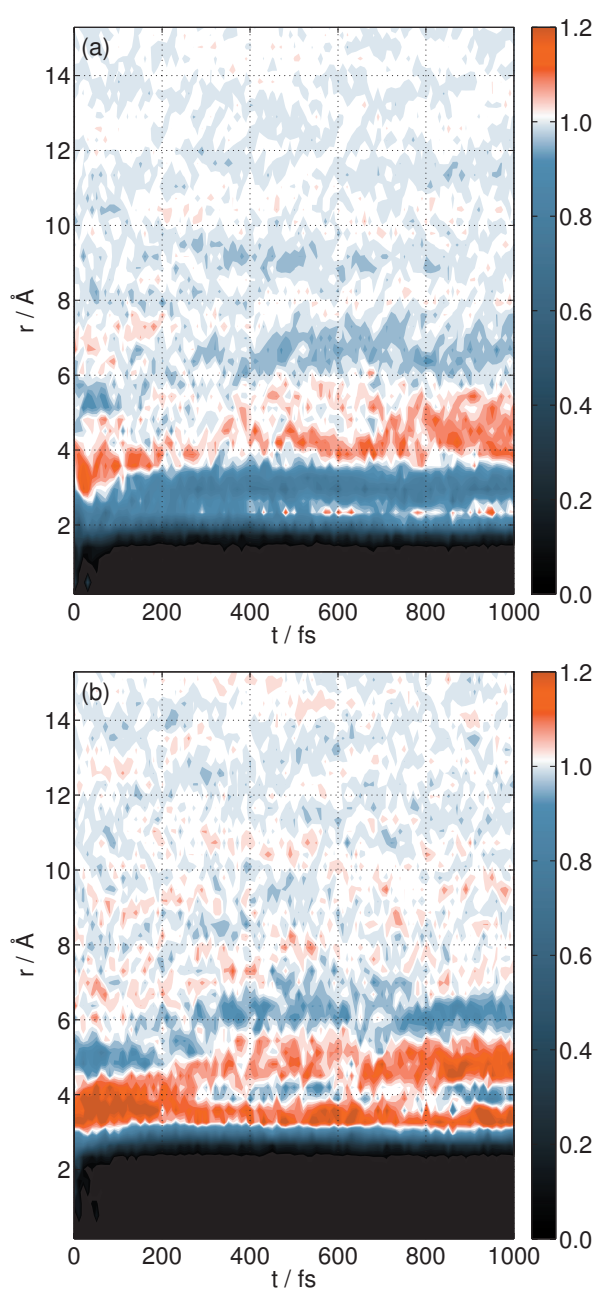

FIG. 11. Solvent dynamics following cold electron injection into water. (a) The time-resolved electron-hydrogen radial distribution function $\left\langle g_{\mathrm{e}-\mathrm{H}}(r, t)\right\rangle$. (b) The time-resolved electron-oxygen radial distribution function $\left\langle g_{\mathrm{e}-\mathrm{O}}(r, t)\right\rangle$.

timescale, $\left\langle g_{\mathrm{e}-\mathrm{H}}(r, t)\right\rangle$ gains a new peak at approximately $2 \AA$ that corresponds to water $\mathrm{OH}$ bonds pointed toward the localized electron. Once the solvent relaxation is complete, at timescales beyond 1 ps, the radial distribution plots in Fig. 11 are entirely consistent with those previously reported from equilibrium simulations. ${ }^{18,63}$ Comparison of Fig. 11 with the corresponding results for helium in Figs. 4(a) and 6(a) emphasizes that water introduces new features during electron localization associated with hydrogen bonding reorientation on the $300 \mathrm{fs}$ timescale.

Another difference between water and helium is that the water dynamics in Fig. 11 does not exhibit the pronounced solvent compression wave that was observed as the excess electron localizes in the helium solvent. This is explained by the fact that water creates the solvent cavity on short timescales by primarily undergoing rotational motions of the individual molecules (Fig. 11), whereas the helium atoms in the vicinity of the localizing electron undergo significant translational motions on short timescales (Fig. 4). These rapid translational impulses in the helium solvent, as well as the hard repulsions between the helium atoms, facilitate the initiation and outward propagation of the solvent compression 

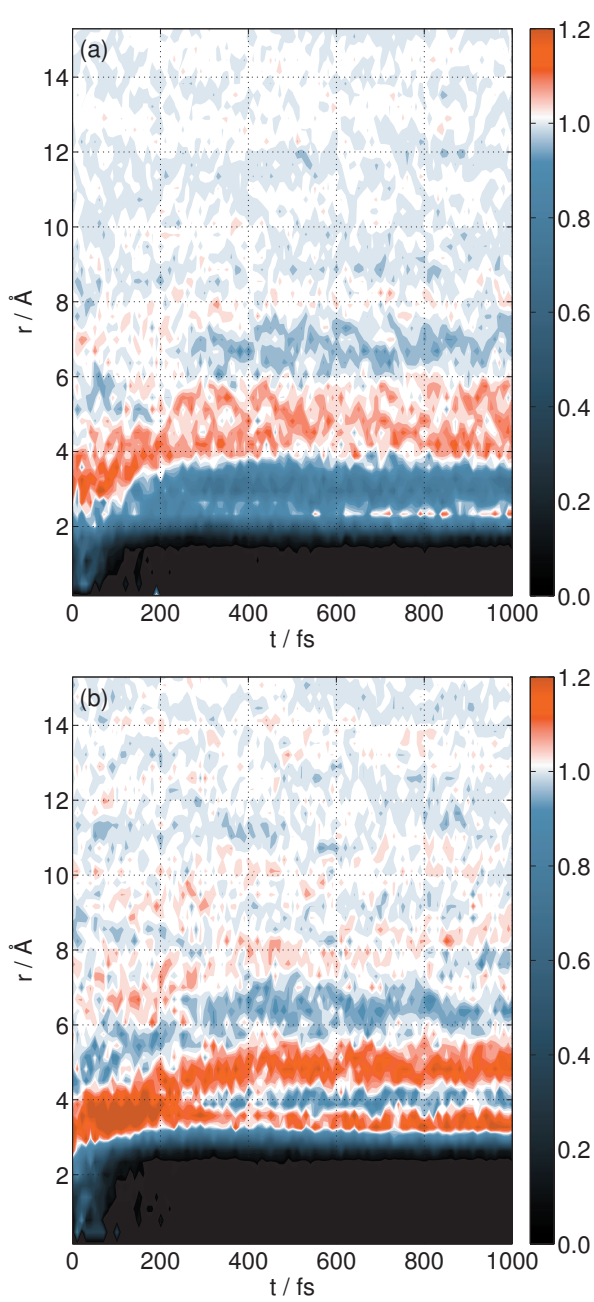

FIG. 12. Solvent dynamics following hot electron injection into water. (a) The time-resolved electron-hydrogen radial distribution function $\left\langle g_{\mathrm{e}-\mathrm{H}}(r, t)\right\rangle$. (b) The time-resolved electron-oxygen radial distribution function $\left\langle g_{\mathrm{e}-\mathrm{O}}(r, t)\right\rangle$.

wave; the absence of translational impulses to the water molecules during electron localization leads to the corresponding absence of the compression wave.

The most prominent difference between the electronsolvent radial distributions for hot (Fig. 12) and cold (Fig. 11) excess electron injection appears at short times. As was observed in the helium simulations, the initial configurations of the electron ring polymer for hot injection are not confined to a single solvent cavity, which leads to an initial nonzero density for the electron-solvent distribution functions at $r=0$, as is consistent with the timescale for ring polymer localization in Fig. 10. At longer times, the electronsolvent distribution functions for hot injection closely resemble those for cold injection.

Figure 13 presents the trajectory-averaged excess electron eigenenergies for cold (blue) and hot (red) electron injection into water. As was seen for helium in Fig. 5, the energies for cold electron injection exhibit a rapid initial decline, with the energy of the ground state dropping by $2.5 \mathrm{eV}$ within the first $30 \mathrm{fs}$ following injection. This initial relaxation timescale is somewhat slower in the hot injection results due to the ring polymer configurations that transiently occupy multiple solvent cavities. A new feature of the water

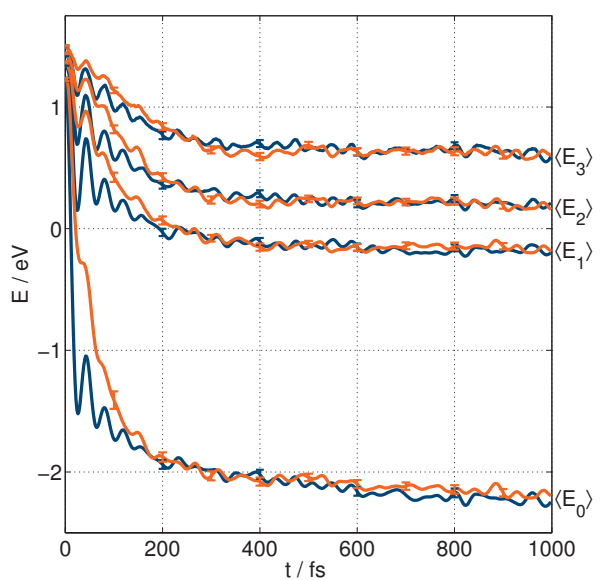

FIG. 13. The four lowest electron eigenenergies, obtained from the nonequilibrium average for RPMD trajectories following cold (blue) and hot (red) injection into the liquid water.

simulations are the oscillations in the eigenenergies on the 30 fs timescale, associated with the librational motion of the water molecules neighboring the excess electron. On the timescales of 200-400 fs, the eigenenergies for both the hot and cold injection simulations continue to relax as distinct water solvent shells appear in Figs. 11 and 12.

In Fig. 14, the transient absorption spectrum for the electron following hot and cold injections are computed using ${ }^{76}$

$$
\begin{aligned}
I(E, t)= & \left\langle\frac{2 \pi}{\hbar} \sum_{n} w(n, t) \sum_{n^{\prime} \neq n}\left|\left\langle\psi_{n}(t)|\mu| \psi_{n^{\prime}}(t)\right\rangle\right|^{2}\right. \\
& \left.\times \delta\left[E-\left(E_{n}(t)-E_{n^{\prime}}(t)\right)\right]\right\rangle,
\end{aligned}
$$

where $\mu$ is the dipole operator, the large angle brackets indicate the nonequilibrium average over the ensemble of

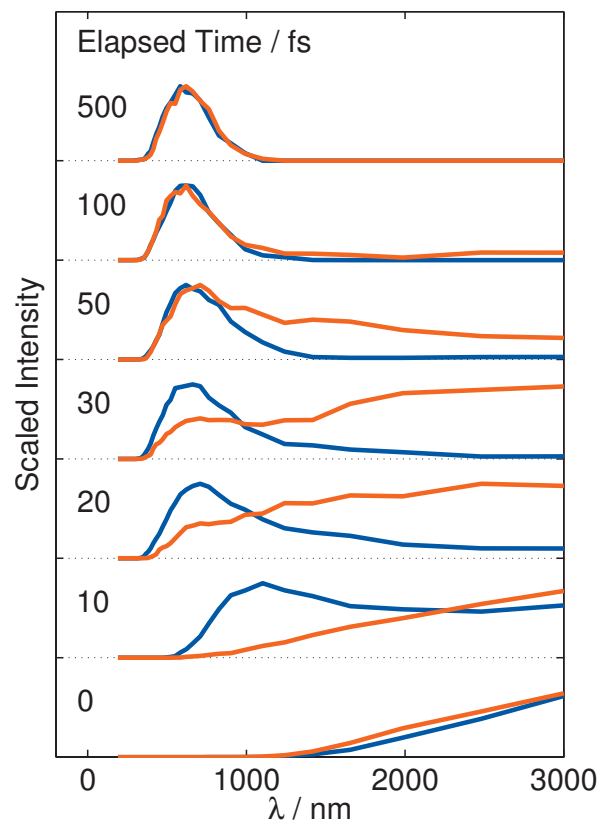

FIG. 14. Transient electron absorption spectra for cold (blue) and hot (red) excess electron injection into water, normalized by the maximum value. Spectra for each waiting time are vertically shifted for clarity. 
RPMD trajectories, and the energy eigenvalues and functions are calculated from the solvent configurations along the trajectories. We employ $w(n, t)=Q(t)^{-1} \exp \left[-E_{n}(t) /\left(k_{\mathrm{B}} T\right)\right]$ to be the instantaneous Boltzmann weights, where $Q(t)=\sum_{n} \exp \left[-E_{n}(t) /\left(k_{\mathrm{B}} T\right)\right]$. Alternative choices for $w(n, t)$, including expressions that account for the overlap between the ring polymer bead positions and the density of the corresponding eigenfunction, do not lead to significant changes in the figure.

Upon initial electron injection, both spectra are necessarily identical within statistical error, featuring a broad absorption band that extends into the infrared. However, the spectrum for cold injection develops an additional peak at 1000 $\mathrm{nm}$ within $10 \mathrm{fs}$. Even though it is clear from Fig. 13 that the electron eigenenergies continue to relax for hundreds of femtoseconds, the ground-to-excited-state energy gaps that dominate the cold electron injection spectrum in Fig. 14 change little after $30 \mathrm{fs}$. The transient absorption spectrum for hot electron injection in Fig. 14 again illustrates the slower relaxation timescale associated with electron localization via multiple solvent cavities. Significant differences between the hot and cold injection trajectories persist for approximately $100 \mathrm{fs}$, after which the electron localization into the ground state is completed and the spectra together approach the equilibrium absorption spectrum.

Many of the features of the RPMD simulations reported here agree well with previous MQCD simulations of electron injection into water. In particular, ground-state simulations of electron injection ${ }^{18}$ find that the excess electron localizes to nearly its equilibrium radius within $30 \mathrm{fs}$, while solvent reordering occurs on a timescale of approximately $200 \mathrm{fs}$. These are the same timescales for cold electron injection obtained from the RPMD model, and as is expected, the transient absorption spectrum for cold electron injection in Fig. 14 is essentially identical to that reported previously. ${ }^{18}$ (We have confirmed that the only significant difference, which appears at $t=0$, is due to the larger system size used in our simulations and the use of a criterion to exclude initially delocalized states in the earlier work. ${ }^{18,77,78}$ ) The slower relaxation timescale observed in the RPMD simulations of hot electron injection is also similar to that found in nonadiabatic MQCD simulations. ${ }^{17}$ These earlier studies found that roughly half of the initially excited electrons relax to the ground state within $50-150$ fs. ${ }^{17}$ Our simulations predict a similar timescale of 30-150 fs for electron localization following hot injection (Fig. 10).

However, in a notable difference from our results, a large fraction of nonadiabatic MQCD trajectories exhibit a timescale of up to $1 \mathrm{ps}$ for the survival of excited electronic states following high-energy injection. ${ }^{16,17,19}$ This slow timescale arises in the MQCD simulations from the slow internal conversion of localized, $p$-type electronic states to the ground state. ${ }^{17,19}$ It is possible that the classical isomorphic model of RPMD simply fails to capture this highly nonadiabatic feature of the collapse dynamics. However, we note that subsequent MQCD simulations that employed a flexible water model find that the slowest nonadiabatic relaxation timescales is approximately 164 fs. Furthermore, ultrafast transient absorption spectra following electron injection also in- dicate that the original $1 \mathrm{ps}$ timescale is too long, with recent results and interpretations finding a timescale for nonadiabatic collapse of less than $150 \mathrm{fs}^{9-11}$ and experimental studies that probe the lifetime of the $p$-type first excited state following photoexcitation of the hydrated electron in water clusters find a short timescale for $p \rightarrow s$ internal conversion of 50 fs. ${ }^{79-81}$

\section{CONCLUSIONS}

We have employed the RPMD model to directly simulate the nonequilibrium injection and relaxation of an excess electron in into supercitical helium fluid and ambient liquid water. A temperature-based method for modulating the initial energy of the excess electron in the RPMD model is presented and used to study both low-energy (cold) and highenergy (hot) electron injections.

For cold injection into fluid helium, the RPMD model is shown to be consistent with electronically adiabatic dynamics with the excess electron in its ground state. The radius of gyration of the ring polymer rapidly approaches its equilibrium value, driving the formation of a solvent compression wave that travels outward and eventually reaches the neighboring periodic replicas of the excess electron. For hot injection into fluid helium, similar solvent dynamics is observed, but the localization of the ring polymer exhibits an additional mechanism for localization via multiple solvent cavities on the timescale of approximately 200 fs. Our comparison of the instantaneous temperature of the solvent and ring polymer beads for the injection simulations in helium illustrates the slow timescale for equilibration between the ring polymer modes and the solvent atoms, as well as simulation artifacts arising from the finite number of ring polymer beads and the finite solvent bath.

For the simulations in liquid water, cold electron injection again leads to the adiabatic localization of the excess electron into a solvent cavity on the timescale of $50 \mathrm{fs}$, whereas hot electron injection leads to a slower relaxation timescale due to metastable ring polymer configurations that occupy multiple solvent cavities. No solvent compression is observed in water because the formation of the solvent cavity involves rotational movements of water molecules rather than rapid translational motion. The water solvent dynamics reveals that distinct first- and second-solvation shells around the electron appear at approximately $300 \mathrm{fs}$ after the initial injection. Our calculation of the transient absorption spectra for the excess electron following injection in water shows clear differences between hot and cold injection at times less than $100 \mathrm{fs}$, but these spectra show little signature of the solvent relaxation dynamics that continues at longer times.

Comparison of the RPMD simulations with prior MQCD simulations reveal broad agreement, both with regard to the observed mechanisms and timescales for solvent and electron relaxation dynamics. Although the RPMD model, along with recent ultrafast spectroscopy studies, does not find evidence for the picosecond-lifetime electronic excited states that are predicted in the MQCD simulations, the similarity between the results obtained with these very different theoretical approaches is encouraging. 


\section{ACKNOWLEDGMENTS}

This work was partially supported by a Camille and Henry Dreyfus Foundation New Faculty Award (to T.F.M.). The authors also thank NERSC for computing resources.

\section{APPENDIX: JUSTIFICATION OF THE ELECTRON INJECTION PROTOCOL}

Here, we provide an alternative justification for the electron injection protocol in Sec. II B by demonstrating that it is closely related to the RPMD approximation to a real-time Kubo-transformed correlation function for which the system is initially in a nonequilibrium distribution.

Suppose that the initial probability distribution in Eq. (4) is replaced with

$$
P_{0}\left(\mathbf{q},\{\mathbf{Q}\} ; T, T^{\prime}\right)=P_{T}(\{\mathbf{Q}\}) \mathcal{P}_{T^{\prime}}(\mathbf{q} \mid\{\mathbf{Q}\}),
$$

where $\mathcal{P}_{T^{\prime}}(\mathbf{q} \mid\{\mathbf{Q}\})$ is the conditional probability distribution for the particle density of the electron,

$\mathcal{P}_{T^{\prime}}(\mathbf{q} \mid\{\mathbf{Q}\})=\frac{\int d \mathbf{q}^{(1)} \cdots d \mathbf{q}^{(n)} \delta\left(\mathbf{q}-\mathbf{q}^{(1)}\right) \exp \left[S\left(\{\mathbf{q}\},\{\mathbf{Q}\} ; T^{\prime}\right)\right]}{\int d \mathbf{q}^{(1)} \cdots d \mathbf{q}^{(n)} \exp \left[S\left(\{\mathbf{q}\},\{\mathbf{Q}\} ; T^{\prime}\right)\right]}$,

rather than the ring polymer centroid density. Then, consider the real-time Kubo-transformed correlation function for which the initial particle density is restricted to be $P_{0}\left(\mathbf{q},\{\mathbf{Q}\} ; T, T^{\prime}\right)$, namely,

$$
\begin{aligned}
\tilde{C}_{A_{0} B}(t)= & \frac{1}{\beta} \int_{0}^{\beta} d \lambda \operatorname{Tr}\left[e^{-\lambda \hat{H}} \hat{A}_{0}(\hat{\mathbf{q}},\{\hat{\mathbf{Q}}\})\right. \\
& \left.\times e^{-(\beta-\lambda) \hat{H}} e^{i \hat{H} t / \hbar} \hat{B}(\hat{\mathbf{q}},\{\hat{\mathbf{Q}}\}) e^{-i \hat{H} t / \hbar}\right],
\end{aligned}
$$

where $\beta=\left(k_{\mathrm{B}} T\right)^{-1}, \hat{H}$ is the Hamiltonian operator for the system, and $\hat{A}_{0}$ and $\hat{B}$ are position-dependent operators, such that

$$
\begin{aligned}
\hat{A}_{0}(\hat{\mathbf{q}},\{\hat{\mathbf{Q}}\})= & \int d \mathbf{q}^{\prime} d \mathbf{Q}_{1}^{\prime} \cdots d \mathbf{Q}_{N}^{\prime} P_{0}\left(\mathbf{q}^{\prime},\left\{\mathbf{Q}^{\prime}\right\} ; T, T^{\prime}\right) \\
& \times \hat{A}(\hat{\mathbf{q}},\{\hat{\mathbf{Q}}\})\left(\delta\left(\mathbf{q}^{\prime}-\hat{\mathbf{q}}\right) \prod_{k=1}^{N} \delta\left(\mathbf{Q}_{k}^{\prime}-\hat{\mathbf{Q}}_{k}\right)\right) .
\end{aligned}
$$

In the limit that the solvent degrees of freedom are treated classically, the RPMD approximation to this correlation function is

$$
\begin{aligned}
\tilde{C}_{A_{0} B}(t)= & \left(\frac{n}{2 \pi \hbar}\right)^{3 n}\left(\frac{1}{2 \pi \hbar}\right)^{3 N} \int d\{\mathbf{q}\} d\{\mathbf{p}\} d\{\mathbf{Q}\} d\{\mathbf{P}\} \\
& \times e^{-\beta H(\{\mathbf{q}\},\{\mathbf{p}\},\{\mathbf{Q}\},\{\mathbf{P}\})} \\
& \times A_{0}\left(\mathbf{q}^{(1)}(0),\{\mathbf{Q}(0)\}\right) B_{n}(\{\mathbf{q}(t)\},\{\mathbf{Q}(t)\}),
\end{aligned}
$$

where

$$
\begin{aligned}
H(\{\mathbf{q}\},\{\mathbf{p}\},\{\mathbf{Q}\},\{\mathbf{P}\})= & \sum_{\alpha=1}^{n} \frac{\left(\mathbf{p}^{(\alpha)}\right)^{2}}{2 m_{n}}+\sum_{j=1}^{N} \frac{\mathbf{P}_{j}^{2}}{2 M_{j}} \\
& +\sum_{\alpha=1}^{n}\left[\frac{1}{2} m_{n} \omega_{n}^{2}\left(\mathbf{q}^{(\alpha)}-\mathbf{q}^{(\alpha+1)}\right)^{2}\right. \\
& \left.+\frac{1}{n} U\left(\mathbf{q}^{(\alpha)},\{\mathbf{Q}\}\right)\right]
\end{aligned}
$$

is the classical Hamiltonian that gives rise to the ring polymer equations of motion [Eqs. (1) and (2)], $\{\mathbf{q}(t)\}$ and $\{\mathbf{Q}(t)\}$ are the positions of the electron ring polymer beads and solvent atoms evolved in time according to these dynamics, $m_{n}=m / n$, and

$$
B_{n}(\{\mathbf{q}\},\{\mathbf{Q}\})=n^{-1} \sum_{\alpha=1}^{n} B\left(\mathbf{q}^{(\alpha)},\{\mathbf{Q}\}\right)
$$

Eq. (A5) can be rearranged to obtain

$$
\begin{aligned}
\widetilde{C}_{A_{0} B}(t)= & \int d \mathbf{q}^{\prime} d \mathbf{Q}_{1}^{\prime} \ldots d \mathbf{Q}_{N}^{\prime} P_{0}\left(\mathbf{q}^{\prime},\left\{\mathbf{Q}^{\prime}\right\} ; T, T^{\prime}\right) \\
& \times \widetilde{C}_{A B}\left(t ; \mathbf{q}^{\prime},\left\{\mathbf{Q}^{\prime}\right\}\right),
\end{aligned}
$$

where

$$
\begin{aligned}
\widetilde{C}_{A B}\left(t ; \mathbf{q}^{\prime},\left\{\mathbf{Q}^{\prime}\right\}\right)= & \left(\frac{n}{2 \pi \hbar}\right)^{3 n}\left(\frac{1}{2 \pi \hbar}\right)^{3 N} \int d\{\mathbf{q}\} d\{\mathbf{p}\} d\{\mathbf{Q}\} d\{\mathbf{P}\} \\
& \times e^{-\beta H(\{\mathbf{q}\},\{\mathbf{p}\},\{\mathbf{Q}\},\{\mathbf{P}\})} \\
& \times\left(A\left(\mathbf{q}^{(1)}(0),\{\mathbf{Q}(0)\}\right) \delta\left(\mathbf{q}^{\prime}-\mathbf{q}^{(1)}(0)\right)\right. \\
& \left.\times \prod_{k=1}^{N} \delta\left(\mathbf{Q}_{k}^{\prime}-\mathbf{Q}_{k}(0)\right)\right) B_{n}(\{\mathbf{q}(t)\},\{\mathbf{Q}(t)\}) .
\end{aligned}
$$

The correlation function in Eq. (A9) corresponds to a system that is initially constrained with respect to the solvent position and the position of one ring-polymer bead. Subject to these constraints, the remaining bead positions and the momenta are sampled from the distribution proportional to $e^{-\beta H(\{\mathbf{q}\},\{\mathbf{p}\},\{\mathbf{Q}\},\{\mathbf{P}\})}$, which corresponds to temperature $T$. RPMD correlation functions of this form are familiar from the flux-side formulation of the RPMD rate theory, where one bead of the ring polymer is initially pinned to a transition state dividing surface. ${ }^{57}$ The full correlation function in Eq. (A8) weights the $\widetilde{C}_{A B}\left(t ; \mathbf{q}^{\prime},\left\{\mathbf{Q}^{\prime}\right\}\right)$ according to the initial particle density distribution $P_{0}\left(\mathbf{q}^{\prime},\left\{\mathbf{Q}^{\prime}\right\} ; T, T^{\prime}\right)$. As for other correlation functions of nonlinear, position-dependent operators, the RPMD approximation in Eq. (A8) is exact to $O\left(t^{3}\right)$ at short times. ${ }^{30}$

The protocol for initializing trajectories to calculate the RPMD correlation function in Eq. (A8) is the same as the protocol put forward in Sec. II B, except that step 3 is modified as follows to account for the difference between the particle density and the centroid density: (3') Keeping both 
the solvent configuration and one bead of the ring polymer fixed, the positions of the remaining ring polymer beads are equilibrated at temperature $T$.

Both the analysis presented in this appendix and the earlier comparison with CMD support the same conclusion: The ring polymer bead positions and velocities that are not determined by the initial distribution $P_{0}\left(\mathbf{q},\{\mathbf{Q}\} ; T, T^{\prime}\right)$ are most naturally sampled at temperature $T$, rather than $T^{\prime}$.

${ }^{1}$ A. Migus, Y. Gauduel, J. L. Martin, and A. Antonetti, Phys. Rev. Lett. 58, 1559 (1987).

${ }^{2}$ H. Lu, F. H. Long, and K. B. Eisenthal, J. Opt. Soc. Am. B 7, 1511 (1990)

${ }^{3}$ X. L. Shi, F. H. Long, H. Lu, and K. B. Eisenthal, J. Phys. Chem. 100, 11903 (1996)

${ }^{4}$ F. H. Long, H. Lu, and K. B. Eisenthal, Phys. Rev. Lett. 64, 1469 (1990).

${ }^{5}$ A. Hertwig, H. Hippler, and A. N. Unterreiner, Phys. Chem. Chem. Phys. 1, 5633 (1999).

${ }^{6}$ A. Hertwig, H. Hippler, and A. N. Unterreiner, J. Phys.: Condens. Matter 12, 165 (2000).

${ }^{7}$ D. Madsen, C. L. Thomsen, J. Thogersen, and S. R. Keiding, J. Chem. Phys. 113, 1126 (2000).

${ }^{8}$ C. R. Wang, T. Luow, and Q. B. Lu, Phys. Chem. Chem. Phys. 10, 4463 (2008).

${ }^{9}$ M. Mizuno, S. Yamaguchi, and T. Tahara, J. Phys. Chem. A 109, 5257 (2005)

${ }^{10}$ V. H. Vilchiz, J. A. Kloepfer, A. C. Germaine, V. A. Lenchenkov, and S. E. Bradforth, J. Phys. Chem. A 105, 1711 (2001).

${ }^{11}$ R. Lian, R. A. Crowell, and I. A. Shkrob, J. Phys. Chem. A 109, 1510 (2005)

${ }^{12}$ T. W. Kee, D. H. Son, P. Kambhampati, and P. F. Barbara, J. Phys. Chem. A $\mathbf{1 0 5}, 8434$ (2001)

${ }^{13}$ P. Kambhampati, D. H. Son, T. W. Kee, and P. F. Barbara, J. Phys. Chem. A 106, 2374 (2002).

${ }^{14}$ R. Laenen, T. Roth, and A. Laubereau, Phys. Rev. Lett. 85, 50 (2000).

${ }^{15}$ C. Pépin, T. Goulet, D. Houde, and J. P. Jay-Gerin, J. Phys. Chem. A 101, 4351 (1997)

${ }^{16}$ F. Webster, P. J. Rossky, and R. A. Friesner, Comput. Phys. Commun. 63, 494 (1991).

${ }^{17}$ F. J. Webster, J. Schnitker, M. S. Friedrichs, R. A. Friesner, and P. J. Rossky, Phys. Rev. Lett. 66, 3172 (1991).

${ }^{18}$ P. J. Rossky and J. Schnitker, J. Phys. Chem. 92, 4277 (1988).

${ }^{19}$ T. H. Murphrey and P. J. Rossky, J. Chem. Phys. 99, 515 (1993).

${ }^{20}$ D. F. Coker and B. J. Berne, J. Chem. Phys. 89, 2128 (1988).

${ }^{21}$ B. Space and D. F. Coker, J. Chem. Phys. 94, 1976 (1991).

${ }^{22}$ B. Space and D. F. Coker, J. Chem. Phys. 96, 652 (1992).

${ }^{23}$ J. Eloranta and V. A. Apkarian, J. Chem. Phys. 117, 10139 (2002).

${ }^{24}$ M. Rosenblit and J. Jortner, J. Phys. Chem. A 101, 751 (1997).

${ }^{25}$ H. D. Meyer and W. H. Miller, J. Chem. Phys. 72, 2272 (1980).

${ }^{26}$ J. C. Tully, J. Chem. Phys. 93, 1061 (1990).

${ }^{27}$ J. C. Tully, Faraday Discuss. 110, 407 (1998).

${ }^{28}$ S. Nielsen, R. Kapral, and G. Ciccotti, J. Chem. Phys. 115, 5805 (2001).

${ }^{29}$ I. R. Craig and D. E. Manolopoulos, J. Chem. Phys. 121, 3368 (2004).

${ }^{30}$ B. J. Braams and D. E. Manolopoulos, J. Chem. Phys. 125, 124105 (2006).

${ }^{31}$ T. F. Miller III, J. Chem. Phys. 129, 194502 (2008).

${ }^{32}$ S. Jang and G. A. Voth, J. Chem. Phys. 111, 2371 (1999).

${ }^{33}$ J. S. Cao and G. A. Voth, J. Chem. Phys. 100, 5106 (1994).

${ }^{34}$ S. M. Jang, Y. Pak, and G. A. Voth, J. Phys. Chem. A 103, 10289 (1999).

${ }^{35}$ R. P. Feynman and A. R. Hibbs, Quantum Mechanics and Path Integrals (McGraw-Hill, New York, 1965).

${ }^{36}$ D. Chandler and P. G. Wolynes, J. Chem. Phys. 74, 4078 (1981).

${ }^{37}$ M. Parrinello and A. Rahman, J. Chem. Phys. 80, 860 (1984).

${ }^{38}$ B. De Raedt, M. Sprik, and M. L. Klein, J. Chem. Phys. 80, 5719 (1984).

${ }^{39}$ N. R. Kestner, J. Jortner, M. H. Cohen, and S. A. Rice, Phys. Rev. 140, A56 (1965).
${ }^{40}$ D. F. Coker, B. J. Berne, and D. Thirumalai, J. Chem. Phys. 86, 5689 (1987).

${ }^{41}$ H. Berendsen, J. P. M. Postma, W. van Gunsteren, and J. Hermans, in Intermolecular Forces, edited by B. Pullman (Reidel, Dordrecht, 1981), p. 331.

${ }^{42}$ J. Schnitker and P. J. Rossky, J. Chem. Phys. 86, 3462 (1987).

${ }^{43}$ L. Turi and D. Borgis, J. Chem. Phys. 117, 6186 (2002).

${ }^{44}$ T. Sommerfeld and K. D. Jordan, J. Phys. Chem. A 109, 11531 (2005).

${ }^{45}$ T. Sommerfeld, A. DeFusco, and K. D. Jordan, J. Phys. Chem. A 112, 11021 (2008).

${ }^{46}$ L. D. Jacobson, C. F. Williams, and J. M. Herbert, J. Chem. Phys. 130, 124115 (2009).

${ }^{47}$ R. E. Larsen, W. J. Glover, and B. J. Schwartz, J. Chem. Phys. 131, 037101 (2009).

${ }^{48}$ J. Schnitker and P. J. Rossky, J. Chem. Phys. 131, 037102 (2009).

${ }^{49}$ X. Y. Chen and S. E. Bradforth, Annu. Rev. Phys. Chem. 59, 203 (2008).

${ }^{50}$ D. Chandler, in Liquids, Freezing and Glass Transition, edited by D. Levesque, J. P. Hansen, and J. Zinn-Justin (Elsevier, New York, 1991), pp. 193-285.

${ }^{51}$ T. D. Hone, P. J. Rossky, and G. A. Voth, J. Chem. Phys. 124, 154103 (2006).

${ }^{52}$ E. Geva, Q. Shi, and G. A. Voth, J. Chem. Phys. 115, 9209 (2001).

${ }^{53}$ W. Smith and T. R. Forester, J. Mol. Graphics 14, 136 (1996).

${ }^{54}$ W. C. Swope, H. C. Andersen, P. H. Berens, and K. R. Wilson, J. Chem. Phys. 76, 637 (1982).

${ }^{55}$ T. F. Miller III and D. E. Manolopoulos, J. Chem. Phys. 123, 154504 (2005).

${ }^{56}$ T. F. Miller III and D. E. Manolopoulos, J. Chem. Phys. 122, 184503 (2005).

${ }^{57}$ I. R. Craig and D. E. Manolopoulos, J. Chem. Phys. 122, 084106 (2005).

${ }^{58}$ I. R. Craig and D. E. Manolopoulos, Chem. Phys. 322, 236 (2006).

${ }^{59}$ T. E. Markland, S. Habershon, and D. E. Manolopoulos, J. Chem. Phys. 128, 194506 (2008).

${ }^{60}$ R. Collepardo-Guevara, I. R. Craig, and D. E. Manolopoulos, J. Chem. Phys. 128, 144502 (2008).

${ }^{61}$ S. Habershon, G. S. Fanourgakis, and D. E. Manolopoulos, J. Chem. Phys. 129, 074501 (2008).

${ }^{62}$ M. Tuckerman, B. J. Berne, and G. J. Martyna, J. Chem. Phys. 97, 1990 (1992).

${ }^{63}$ J. Schnitker and P. J. Rossky, J. Chem. Phys. 86, 3471 (1987).

${ }^{64}$ H. C. Andersen, J. Comput. Phys. 52, 24 (1983).

${ }^{65}$ A. Wallqvist, D. Thirumalai, and B. J. Berne, J. Chem. Phys. 86, 6404 (1987).

${ }^{66}$ B. J. Schwartz and P. J. Rossky, J. Chem. Phys. 101, 6902 (1994).

${ }^{67}$ B. J. Schwartz and P. J. Rossky, J. Chem. Phys. 101, 6917 (1994).

${ }^{68}$ O. Steinhauser, Mol. Phys. 45, 335 (1982).

${ }^{69}$ D. Chandler and K. Leung, Annu. Rev. Phys. Chem. 45, 557 (1994).

${ }^{70}$ P. W. Anderson, Phys. Rev. 109, 1492 (1958).

${ }^{71}$ J. Schnitker, K. Motakabbir, P. J. Rossky, and R. Friesner, Phys. Rev. Lett. 60, 456 (1988).

${ }^{72}$ A. V. Benderskii, J. Eloranta, R. Zadoyan, and V. A. Apkarian, J. Chem. Phys. 117, 1201 (2002).

${ }^{73}$ R. W. Hall and B. J. Berne, J. Chem. Phys. 81, 3641 (1984).

${ }^{74}$ J. Schnitker, P. J. Rossky, and G. A. Kenneywallace, J. Chem. Phys. 85, 2986 (1986).

${ }^{75}$ K. A. Motakabbir, J. Schnitker, and P. J. Rossky, J. Chem. Phys. 97, 2055 (1992)

${ }^{76}$ B. J. Berne and R. Pecora, Dynamic Light Scattering (Dover, Mineola, NY, 2000).

${ }^{77}$ K. A. Motakabbir and P. J. Rossky, Chem. Phys. 129, 253 (1989).

${ }^{78}$ Smaller differences between our spectra and those reported in Ref. 18 are attributed to the method of calculating the eigenvalues. We have confirmed that our electron absorption spectrum for water at equilibrium quantitatively matches the more recently reported results in Ref. 16.

${ }^{79}$ A. E. Bragg, J. R. R. Verlet, A. Kammrath, O. Cheshnovsky, and D. M. Neumark, Science 306, 669 (2004).

${ }^{80}$ A. E. Bragg, J. R. R. Verlet, A. Kammrath, O. Cheshnovsky, and D. M. Neumark, J. Am. Chem. Soc. 127, 15283 (2005).

${ }^{81}$ O. T. Ehrler and D. M. Neumark, Acc. Chem. Res. 42, 769 (2009). 